\title{
Utilização de nanopartículas de ferro zero na degradação do corante reativo RED 243
}

\author{
Zero valent iron nanoparticles using the deterioration of dye reactive RED 243 \\ Felipe Sombra Santos*, Lidia Yokoyama, Fabiana Valeria Fonseca
}

Como citar esse artigo. Santos FS, Yokoyana L, Fonseca FV. Utilização de nanopartículasdeferrozeronadegradação do corante reativo RED 243 Revista Teccen. 2016 Jul./Dez.; 09 (2): 03-12.

\begin{abstract}
Resumo
A utilização de nanopartículas de ferro de valência zero (nZVI) empregadas na degradação de um corante reativo foi avaliado neste trabalho. O ferro elementar utilizado foi caracterizado pela técnica de difração de raios-X (DR-X), e os picos de difração foram obtidos nos ângulos de $44,75^{\circ}, 65,1^{\circ}$ e $82,5^{\circ}$. O primeiro ângulo estava associado ao ferro elementar com estrutura cristalina de cubo de corpo centrado, com maior intensidade e para os demais ângulos foram observados a mesma fase, em uma menor proporção intensidade de contagem. Os ensaios foram realizados, em valores de temperatura e $\mathrm{pH}$ estabelecidos.Os resultados obtidos mostraram que ao utilizar uma concentração de $20 \mathrm{mg} . \mathrm{L}^{-1}$ do corante e uma massa de 20 e 50 mg de nZVI, tanto em valor de pH igual a 2,0 ou 3,0, foi possível notar a degradação do corante, em função do tempo. As constantes cinéticas de degradação do corante foram obtidas a partir dos ensaios em temperaturas de $25,35,45$ e $50{ }^{\circ} \mathrm{C}$, para uma massa de nZVI igual a $20 \mathrm{mg}$; concentração do corantede $20 \mathrm{mg} . \mathrm{L}^{-1}$; pH ajustado e mantido em 3,0 com uma velocidade de agitação de $150 \mathrm{rpm}$. A máxima degradação do corante ocorreu após 70 minutos de experimento, para uma concentração de $20 \mathrm{mg} . \mathrm{L}^{-1}$ de corante, massa de nZVI utilizada de $20 \mathrm{mg}$ e o pH ajustado em 3,0, possibilitando assim, atingir uma eficiência de degradação superior a 80\%. A partir dos dados cinéticos obtidos, foi possível determinar a energia de ativação do sistema, sendo este valor igual a $25,8 \mathrm{~kJ} \mathrm{~mol}^{-1}$, sugerindo um mecanismo difusional ao sistema. AsnZVI empregadas na degradação do corante permitiram observar que o processo ocorreu através da transferência de elétrons, em meio ácido, no qual o ferro se oxidava e a estrutura do corante se reduzia, ao longo do tempo.
\end{abstract}

Palavras-Chave: Nanopartículas de ferro de valência zero; Degradação; Corante reativo; cinética.

\begin{abstract}
The use of nanoparticles of zero valent iron (nZVI) employed in the degradation of a reactive dye was evaluated in this work. The nZVI used was characterized by the technique of X-ray diffraction (X-DR), and diffraction peaks were obtained at angles of $44.75^{\circ}, 65.1^{\circ}$ and $82.5^{\circ}$. The first angle was associated with elemental iron crystal structure of body-centered cube, with higher intensity and for the other angles were observed at the same phase in a minor proportion count intensity. The tests were performed at temperatures and $\mathrm{pH}$ values established. The results showed that by using a dye concentration of $20 \mathrm{mg} . \mathrm{L}^{-1}$ and a mass of 20 and $50 \mathrm{mg}$ of nZVI in both pH value equal to 2.0 or 3.0 , it was possible to notice the degradation of dye by the time. The kinetic constants of dye degradation were obtained from the testing temperatures of $25,35,45$ and $50{ }^{\circ} \mathrm{C}$, for a nZVI mass equal to $20 \mathrm{mg}$; dye concentration of $20 \mathrm{mg} . \mathrm{L}^{-1} ; \mathrm{pH}$ adjusted and maintained at 3.0, with a stirring speed of $150 \mathrm{rpm}$. Maximum degradation of the dye occurred after 70 minutes of the experiment at a concentration of $20 \mathrm{mg} . \mathrm{L}^{-1}$ dye used, nZVI mass of $20 \mathrm{mg}$ and the $\mathrm{pH}$ adjusted to 3.0, thus enabling to achieve a higher degradation efficiency $80 \%$. From the kinetic data obtained, it was possible to determine the system's activation energy, and this value of $25.8 \mathrm{~kJ} \mathrm{~mol}^{-1}$, suggesting a diffusion mechanism to the system. The nZVI employed in dye degradation allowed the observation that the process occur through electron transfer, in acid medium, in which iron nanoparticle is oxidized and the dye reactive structure is reduced over time.
\end{abstract}

Keywords: Nanoparticles of zero valent iron; Degradation; Reactive dye, Kinetics.

\section{Introdução}

A remoção da cor dos efluentes de origem têxtil é um dos problemas ambientais existentes, por ser necessária a utilização de tratamentos mais avançados que permitam promover a remoção de um poluente recalcitrante, pois o tratamento convencional, não é suficiente para inibir o grau de poluição e conseguir degradar uma determinada substância no ambiente (Borba et al., 2012; Braile, 1971; Cavalcanti, 2009;
Marin et al., 2015; Metcalf \& Eddy, 2016; Módenes et al., 2011, Nunes, 2008).

A vantagem da utilização de nZVI, quando comparadas as partículas de tamanho micrométricas, se deve a boa eficiência durante as reações de redução, na alta reatividade, e por apresentar uma elevada área superficial, permitindo uma melhor mobilidade da molécula. Ainda assim, as partículas de ferro em tamanho de escala nanométrica permanecem em suspensão por um longo intervalo de tempo, facilitando as diversas

Afiliação dos autores: Universidade Severino Sombra, Vassouras-RJ, Brasil.

* Endereço para correspondência: Universidade Severino Sombra, Av. Exped. Oswaldo de Almeida Ramos, 280 - Centro - Vassouras, RJ - CEP $27700-000$.

email: fpsombra@ig.com.br 
aplicações conhecidas, voltadas para remediação do solo e tratamento de efluentes (Elliot, 2001).

Uma das aplicações do ferro na forma elementar pôde ser observada, através da desalogenação redutiva, no qual o ferro é usado na forma de barreira reativa, por exemplo. Essas barreiras citadas por serem permeáveis criam uma área de tratamento reativa, capaz de interceptar a pluma contaminada e removendo "in loco" a pluma contaminada, através de processos físicos, químicos e biológicos (Arruda, 2005).

Segundo Nurmi (2005), a síntese de nZVI pode ser obtida por diferentes metodologias, como via hidrogenação (Zhang, 2011), decomposição térmica (Karlsson, 2005), via oxidação do hidrogênio gasoso (Kim, 2009) e via reação com borohidreto de sódio (Soeira, 2007; Souza, 2007; Sun, 2006; Sun, 2007).

A degradação de um corante reativo através do uso de nanopartículas de ferro de valência zero, em contato direto com a solução do corante, em diferentes concentrações e condições experimentais, a fim de observar a eficiência de remoção do corante ao longo do tempo e obter os dados cinéticos do processo foram avaliados nesse trabalho.

\section{Material e métodos}

As nanopartículas de ferro de valência zero utilizadas nos ensaios foram obtidas através da mKNANO (Ontário, Canadá). As partículas apresentavam um diâmetro médio de $25 \mathrm{~nm}$. Ocorante reativo usado foi o vermelho Drimaren X-6BN 150 (C.I. ReactiveRed243), fornecidos pela Clariant. Este corante apresenta na fórmula molecular, estruturas de cloretocianurilo (monoclorotriazina), como bases reativas. A fórmula molecular do corante empregado é apresentada pela Figura 1.

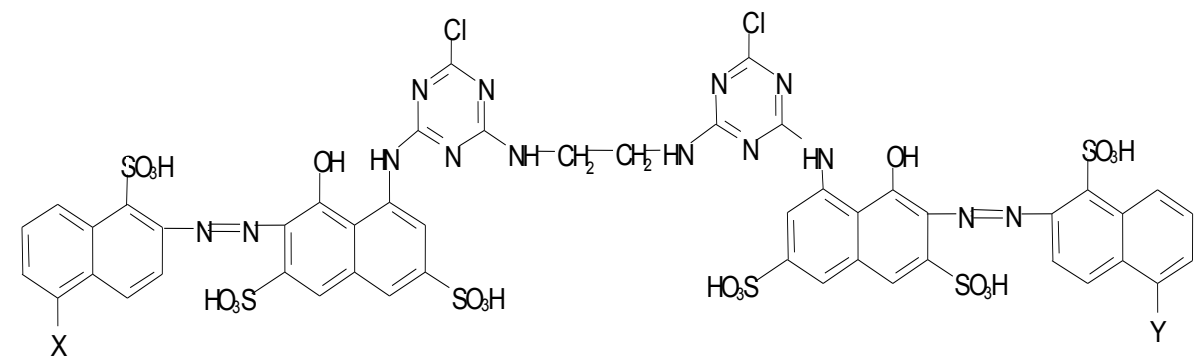

Figura 1. Fórmula estrutural do corante azo Vermelho Drimaren X-6BN.

O corante utilizado é, na realidade, uma mistura de três tipos de corantes. No corante do tipo 1, as estruturas representadas por $\mathrm{X}$ e $\mathrm{Y}$ são substituídos pelos grupos funcionais $\mathrm{SO}_{3} \mathrm{H}$. Já para o segundo tipo de corante, o radical X é substituído por $\mathrm{SO}_{3} \mathrm{H}$ enquanto que, o Y por $\mathrm{H}$. E finalmente, na estrutura do corante do tipo 3, X e Y são substituídos pelo hidrogênio. Partindo da suposição de que os três corantes usados estejam em proporções molares iguais, a massa molecular média da mistura dos três tipos de corante é em torno de $1.469,57 \mathrm{mg} \cdot \mathrm{mol}^{-1}$. (Colour Index).

Os ensaios de degradação do corante, foram realizados com quantidades de 9,20 e $50 \mathrm{mg}$ de nZVI para um volume de $100 \mathrm{~mL}$. A concentração do corante usada foi de $20 \mathrm{mg} \cdot \mathrm{L}^{-1}$. A faixa de $\mathrm{pH}$ analisada foi de $2 \mathrm{e}$ 3 , pois nesses valoresas nanopartículas junto ao corante poderão ser melhor avaliadas, pois há uma tendência de ocorrência de reação e lixiviação, conforme pode ser observada pelo diagrama de predominância do ferro, ilustrado na Figura 2. E o valor de $\mathrm{pH}$ igual 7 usado, foi devido a região da neutralidade.

Uma quantidade de nanopartículas de ferro necessária ao experimento foi pesada e depois transferida para um recipiente contendo $100 \mathrm{~mL}$ da solução de corante, no qual o $\mathrm{pH}$ e a temperatura eram ajustados e mantidos constantes durante todos os ensaios. O conjunto era colocado em uma mesa agitadora regulada para uma velocidade de 150 rpm, e a cada 10 minutos, era retirada uma aliquota da solução para avaliar a coloração do corante, o $\mathrm{pH}$ e a temperatura. Elas eram então filtradas em membranas de $0,45 \mu \mathrm{m}$, e em seguida, analisadas em um espectrofotômetro.

A concentração do corante ao longo do tempo foi avaliada em um espectrofotometro Shimadzu, modelo UV-Mini - 1240 no comprimento de onda de $517 \mathrm{~nm}$. Enquanto que a concentração de ferro total, foi analisada através de espectrofotômetro DR 2800 (Hach), usando a programação para FerroVer (método 265 do aparelho). Os ajustes de $\mathrm{pH}$ foram feitos através de soluções de $\mathrm{HCl}$ e de $\mathrm{NaOH}$, conforme o valor de $\mathrm{pH}$ para cada experimento.

A concentração de peróxido de hidrogênio foi avaliada por método colorimétrico (Mattos et al, 2003; Oliveira et al, 2001), com auxílio do espectrofotômetro $\mathrm{HACH}$, modelo DR-2800, ajustado para o comprimento de onda de $460 \mathrm{~nm}$, no qual é possível observar a formação do cátion peroxovanádio (V) que apresenta uma coloração avermelhada. $\mathrm{O}$ procedimento da análise consiste em construir uma curva $\left[\mathrm{H}_{2} \mathrm{O}_{2}\right] \mathrm{x}$ Absorbância, através da adição de $4 \mathrm{~mL}$ de amostra com 1,6 mL de metavanadato de amônio e completar 


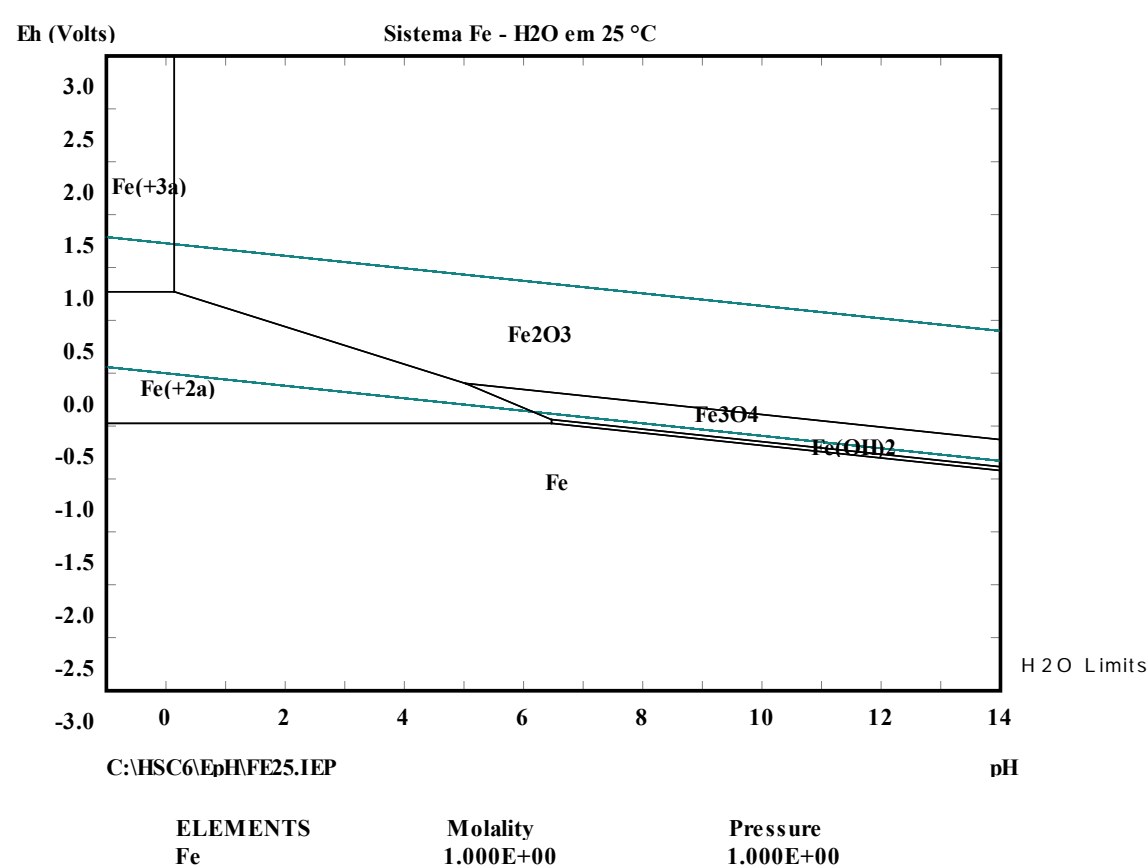

Figura 2. Diagrama Eh - pH do sistema $\mathrm{Fe}-\mathrm{H} 2 \mathrm{O}$ a $25^{\circ} \mathrm{C}$ (Roine, 2006)

o volume até $10 \mathrm{~mL}$ com água deionizada. A solução de metavanadato de amônio é preparada utilizando 1,17 $\mathrm{g}$ de $\mathrm{NH}_{4}(\mathrm{VO})_{3}$ em $5,56 \mathrm{~mL}$ de ácido sulfúrico $9,0 \mathrm{M}$ e completando o volume da solução até $100 \mathrm{~mL}$, com água deionizada, gerando uma concentração final de metavanadato de amônio de 0,1 M. Segundo Mattos et al. (2003) comportamento estequiométrico do peróxido de hidrogênio é ilustrado, através da equação (1), que representa o comportamento do agente oxidante, na presença de metavanadato de amônio.

$$
\mathrm{VO}^{-3}+\mathrm{HH}^{+}+\mathrm{H}_{2} \mathrm{O}_{2} \rightarrow \mathrm{VO}_{2}^{3}+3 \mathrm{H}_{2} \mathrm{O}
$$

\section{Resultados e discussão}

\section{Caracterização das nanopartículas de ferro de valência zero}

O ensaio de caracterização das nanopartículas de ferro de valência zero foi realizado em um Difratômetro RigakuMiniFlex II, operado a $30 \mathrm{kV}$ e $15 \mathrm{~mA}$. A varredura foi realizada em um intervalo de ângulo de 5 a $90^{\circ}$, com passo de $0,5^{\circ}$. A identificação dos picos obtidos se deu através do auxílio do Materials Data Jade5 (5.0.37) XRD PatternProcessing Serial MDI-R99100.

A Figura 3 apresenta o difratograma característico do ferro de valência zero, onde pode-se observar que os picos de difração ocorreram para os ângulos de $44,75^{\circ}, 65,1^{\circ}$ e $82,5^{\circ}$, considerando que a maior reflexão ocorreu em $44,75^{\circ}$. Esses ângulos

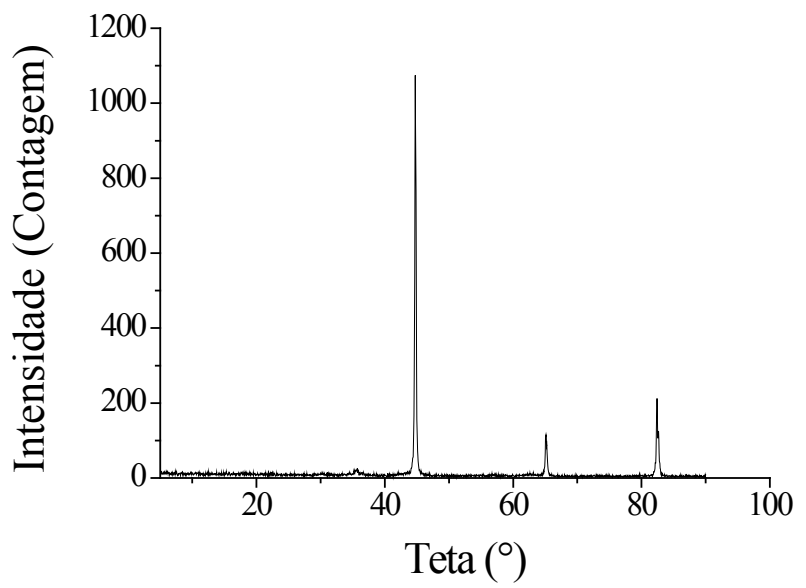

Figura 3. Difratograma das nanopartículas de ferro de valência zero 
estão associados à estrutura cristalina de cubo de corpo centrado do ferro elementar. Tais valores dos ângulos de difração mencionados também são citados em outras literaturas existentes. (Kim, 2009; Lin, 2010; Sun, 2006)

\section{Avaliação da degradação do corante}

Inicialmente foi experimentado uma quantidade de $9 \mathrm{mg} . \mathrm{L}^{-1}$ de nZVI, conforme previsto na literatura (Sun et al., 2013) para uma concentração de corante de 100 mg.L-1 (Araujo, 2008), sob agitação de $150 \mathrm{rpm}$, sem ajuste do $\mathrm{pH}$.

Foi observado que para uma concentração de 100 mg. $L^{-1}$ de corante, era necessário realizar sucessivas diluições das amostras, para realização da leitura no espectrofotômetro, o que poderia acarretar um aumento no erro experimental. Em vista disso, o mesmo teste foi conduzido com uma concentração de $20 \mathrm{mg} . \mathrm{L}^{-1}$ de corante. Os resultados dos ensaios mencionados, podem ser visualizados pela Figura 4.

Pela Figura 4 é possível observar que em nenhuma das concentrações do corante apresentada, promoveu-se a degradação do corante. Tal fato já era esperado, pois como o $\mathrm{pH}$ do sistema não foi ajustado para o meio ácido, as nanopartículas de ferro elementar que provavelmente estavam em pouca quantidade, não se lixiviaram (Figura 2), e consequentemente, não reagiram com o corante em solução, não permitindo assim, a degradação desejada. Durante este ensaio, o $\mathrm{pH}$ da amostra, ao longo do tempo, permaneceu praticamente em 6,02 e 6,11, para as concentrações de 20 e $100 \mathrm{mg}^{-\mathrm{L}^{-1}}$ de corante, respectivamente.

Um outro ensaio foi experimentado, praticamente nas mesmas condições anterior, todavia com uma quantidade de $20 \mathrm{mg}$ de nZVI incorporados ao sistema, para diferentes concentrações de corante, sem ajuste do $\mathrm{pH}$, a fim de verificar uma possível degradação do corante em estudo. Os resultados obtidos desse ensaio são apresentados na Figura 5.

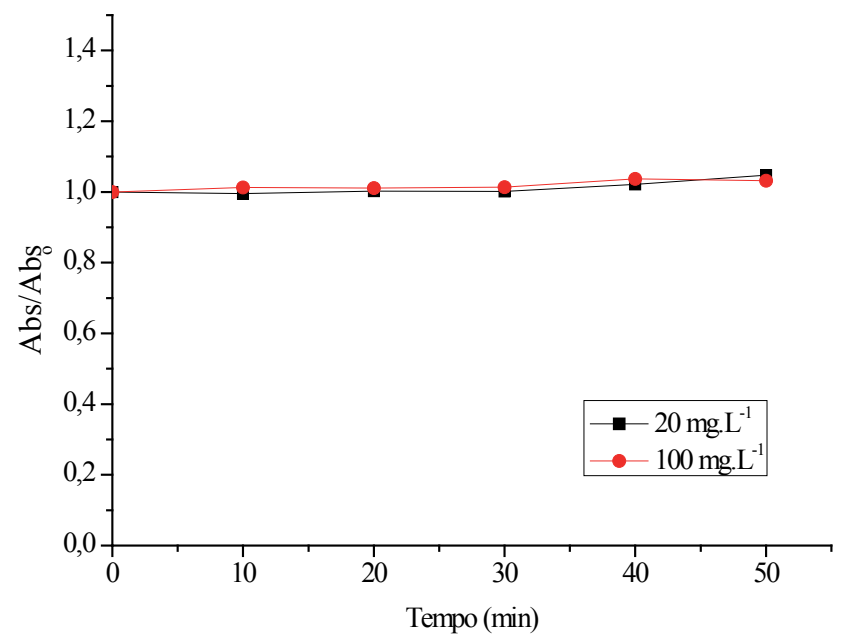

Figura 4. Perfil da degradação do corante ao longo do tempo com uma massa de $9 \mathrm{mg}$ de nZVI.Condição ensaiada: $\mathrm{Fe}=9 \mathrm{mg} \cdot \mathrm{L}-1 ; \mathrm{T}=25^{\circ} \mathrm{C}$; sem ajuste de $\mathrm{pH}$ e $150 \mathrm{rpm}$.

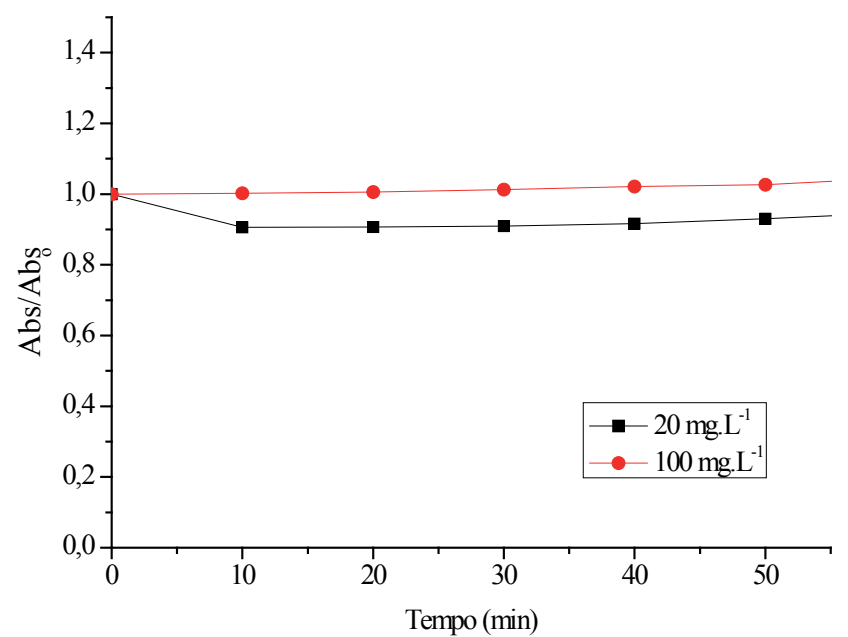

Figura 5. Perfil da degradação do corante ao longo do tempo com uma massa de $20 \mathrm{mg}$ de nZVI. Condição ensaiada: $\mathrm{Fe}=200 \mathrm{mg} \cdot \mathrm{L}^{-1} ; \mathrm{T}=25^{\circ} \mathrm{C}$; sem ajuste de $\mathrm{pH}$ e $150 \mathrm{rpm}$ 
Como a degradação do corante não ocorria em um sistema, sem ajuste de $\mathrm{pH}$, realizou-se então um ensaio em pH igual a 2,0, com diferentes quantidades de nZVI, e uma concentração de corante de $20 \mathrm{mg} \cdot \mathrm{L}^{-1}$. Os resultados desse ensaio estão expostos na Figura 6.

Com os resultados apresentados pela Figura 6 ficou evidente que a degradação só iria ocorrer, se o sistema fosse acidificado. A diferença entre a quantidade mássica de nZVI de $20 \mathrm{mg}$ e $50 \mathrm{mg}$, na solução não promoveu uma diferença muito significativa na cinética de degradação do corante ao longo do ensaio, que pudesse justificar a utilização de $50 \mathrm{mg}$ para os demais ensaios, ao invés de $20 \mathrm{mg}$ de nZVI. Tal justificativa também se comprovou na literatura, quando se utilizou na condição ótima de ensaio, $20 \mathrm{mg}$ de nZVI, durante um período de 60 minutos, para degradar um outro tipo de corante ensaiado, na concentração de $0,3 \mathrm{mM}$ (Moon et al., 2011). Ainda analisando a Figura 6 e fazendo uma comparação entre a eficiência de degradação do corante em meia hora de experimento, nas duas curvas apresentadas, foi possível constatar que 39,90 e $51,29 \%$ do corante foram degradados para as respectivas quantidades de 20 e $50 \mathrm{mg}$ de nZVI. Todavia, quando foi completada uma hora de experimento as eficiências de remoção obtidas para as mesmas quantidades de nZVI foram 67,00 e 74,01\% de degradação, respectivamente. Sendo assim, mais uma vez não se justificou a utilização de $50 \mathrm{mg}$ de nZVI, pois a degradação não obedeceu a proporção mássica utilizada nos ensaios. A Figura 7, apresenta as eficiências de remoção mencionadas.

Ao fixar a concentração do corante em 20 mg. $\mathrm{L}^{-1}$ e a massa das nanopartículas em $20 \mathrm{mg}$, e para

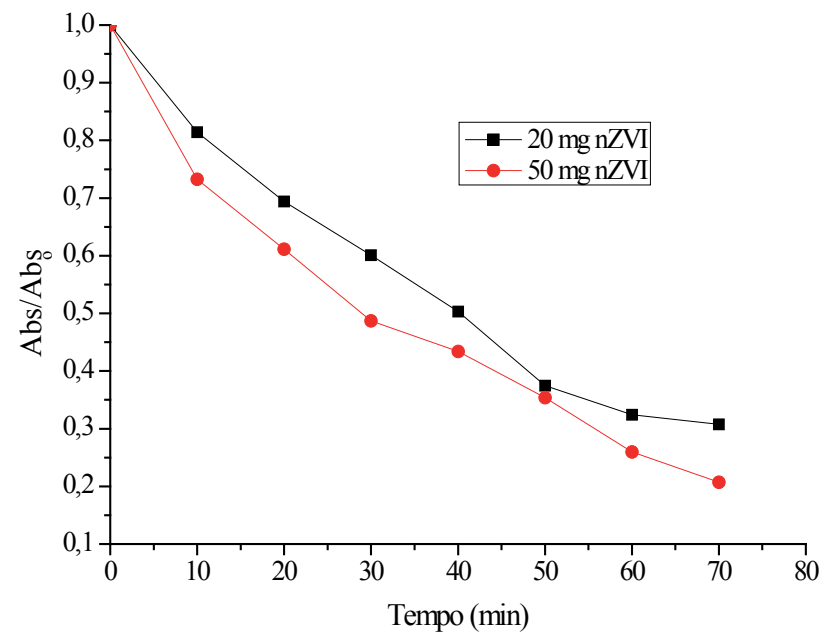

Figura 6. Perfil da degradação do corante ao longo do tempo na concentração de $200 \mathrm{mg}$.L-1 de corante. Condição ensaiada: $\mathrm{Fe}=20 \mathrm{mg}$; [corante] $=20 \mathrm{mg}$.L-1; T $=25^{\circ} \mathrm{C} ; \mathrm{pH}=2,0$ e $150 \mathrm{rpm}$

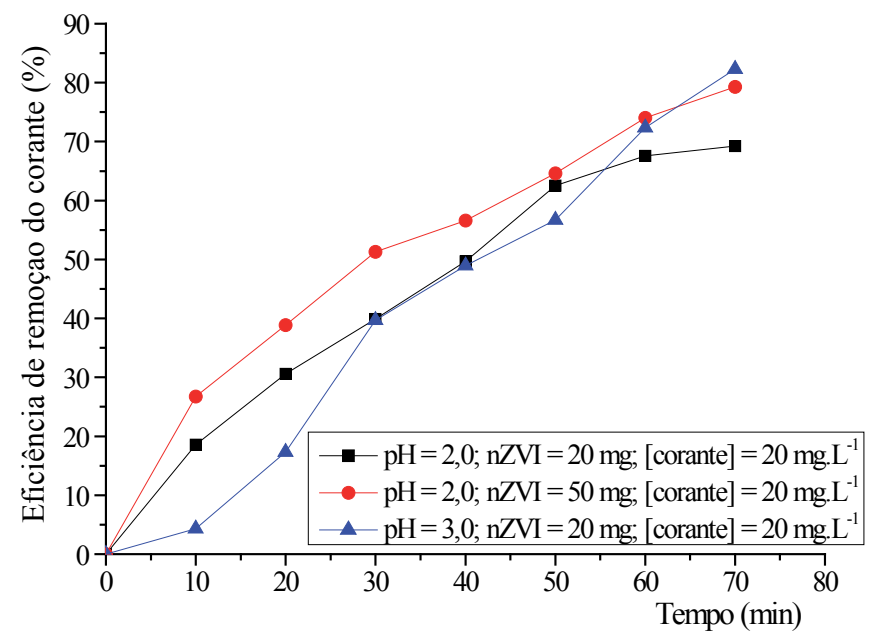

Figura 7. Perfil da eficiência de remoção do corante em diferentes condições experimentais ao longo do tempo.Condição ensaiada: $\mathrm{T}=25^{\circ} \mathrm{C}$ e $150 \mathrm{rpm}$ 
diferentes valores de $\mathrm{pH}$, foi possível presenciar um comportamento diferenciado, quando buscou comparar o $\mathrm{pH}$, conforme pode ser notado na Figura 8. A curva no qual não houve ajuste de $\mathrm{pH}$, foi realizado no $\mathrm{pH}$ da amostra que foi igual a 6,5 .
Através da Figura 8 foi possível de constatar que a degradação em valor de $\mathrm{pH}$ igual a 3,0 foi praticamente a mesma, quando comparada para o valor de $\mathrm{pH}$ igual a 2,0 . O coeficiente de ajuste da curva para $\mathrm{pH}$ igual a 3,0, teve um melhor ajuste $\left(\mathrm{R}^{2}=0,9944\right)$, quando comparado

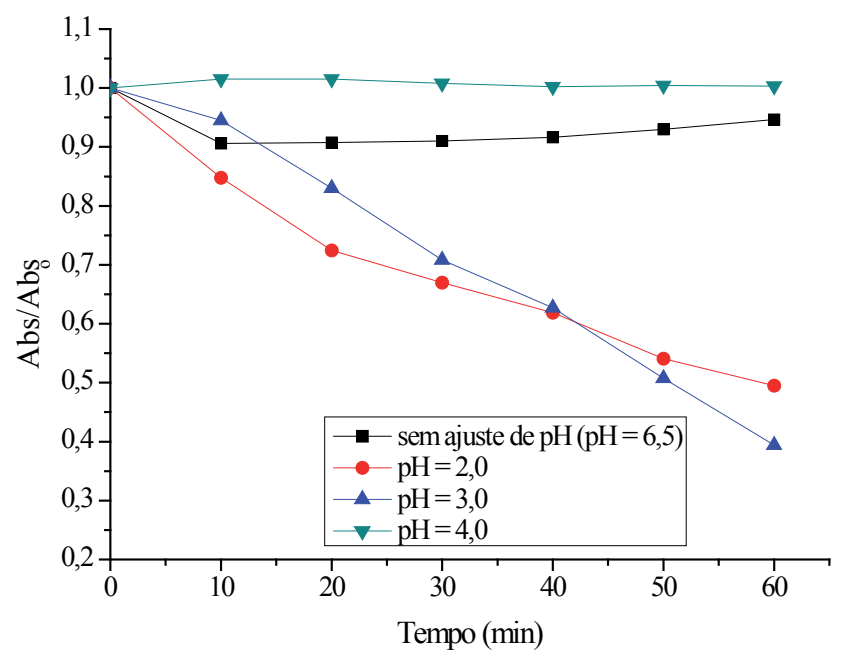

Figura 8. Perfil de degradação do corante em diferentes valores de $\mathrm{pH}$ ao longo do tempo.Condição ensaiada: $\mathrm{Fe}=200 \mathrm{mg} . \mathrm{L}-1$; [corante] $=20 \mathrm{mg} . \mathrm{L}-1 ; \mathrm{T}=25^{\circ} \mathrm{C}$ e 150 rpm.

ao $\mathrm{pH}$ igual a 2,0, cujo o valor de $\mathrm{R}^{2}$ foi igual a 0,9518 . Tais sistemas ácidos promoveram a degradação desejada, conforme previsto pela Figura 2. E para os demais ensaios observados não ocorreu degradação do corante. A degradação do corante em meio ácido se justificou pela lixiviação do ferro elementar, que foi transformado na forma iônica (Figura 2), promovendo a oxidação do metal, permitindo a liberação dos elétrons do metal no sistema, que foram utilizados na redução da estrutura molecular do corante.

Em outros trabalhos publicados na literatura (Joo et al., 2004; Keenan e Sedlak, 2008; Lee e Sedlak, 2008; Xia et al., 2014) foram estudados a degradação de compostos orgânicos (metanol e herbicidas), utilizando nZVI, sem a presença de peróxido de hidrogênio. Neles a quebra da estrutura do composto orgânico ocorria devido a oxidação do ferro em meio ácido, que na presença de oxigênio, gerou como um subproduto, o peróxido de hidrogênio no sistema. Essa substância oxidante gerada, quando em contato com o íon ferroso, promovia a geração do radical hidroxila, e que, consequentemente, permitia a ocorrência da reação de Fenton dentro do sistema. As reações (2-4) sugerem o mecanismo reacional do processo descrito.

$$
\mathrm{Fe}_{\mathrm{C}_{1}}+\mathrm{O}_{2}+2 \mathrm{H}^{4} \rightarrow \mathrm{H}_{2} \mathrm{O}_{2}+\mathrm{Fe}^{2+}
$$

$$
\begin{aligned}
& \mathrm{Fe}^{2+}+\mathrm{H}_{2} \mathrm{O}_{2} \rightarrow \mathrm{Fe}^{3+}+\mathrm{OH}+\mathrm{OH}^{-} \\
& 2 \mathrm{Fe}^{2+}+\mathrm{Fe}^{0} \rightarrow 3 \mathrm{Fe}^{2+}
\end{aligned}
$$

Conforme exposto nas reações (2) a (4), e prevendo a formação de peróxido de hidrogênio, assim como, a ocorrência da reação de Fenton, a temperatura reacional mantida controlada $\left(25^{\circ} \mathrm{C}\right)$ no sistema e o equipamento no qual o sistema permaneceu sob agitação constante, era vedado, não permitindo o contato com o meio externo, minimizando assim,os possíveis interferentes. A quantificação da concentração de peróxido residual foi então avaliada, pela reação do peróxido de hidrogênio com o metavanadato de amônio, conforme descrito na metodologia. O ensaio de degradação do corante foi realizado, sem a utilização do peróxido de hidrogênio como um reagente. $\mathrm{O}$ pH estava ajustado em 3,0 , a concentração do corante era de $20 \mathrm{mg} . \mathrm{L}^{-1} \mathrm{e}$ a massa de $20 \mathrm{mg}$ de nZVI. Os resultados obtidos para este ensaio foram apresentados na Figura 9, na qual as variáveis mensuradas foram avaliadas em função do tempo de reação.

Pela Figura 9 foi possível de observar que, não foi constatada a presença do peróxido de hidrogênio residual 
no sistema, durante a degradação da concentração do corante, conforme descrito anteriormente. Isso não significa dizer que não houve a possibilidade de geração de peróxido de hidrogênio ao sistema, durante o ensaio, conforme observado pelas reações (2-4) e o total consumo deste reagente oxidante durante o experimento, pois a metodologia empregada só avalia a concentração de peróxido residual.
Com os resultados obtidos nos ensaios em diferentes valores de $\mathrm{pH}$, apresentados pela Figura 8, foi possível plotar uma outra relação, envolvendo a concentração do corante, em função do tempo, para avaliar a degradação do corante, conforme é apresentado na Figura 10.

Para os valores de $\mathrm{pH}$ iguais a 2 e 3, da Figura 10, foram determinados os coeficientes de ajuste

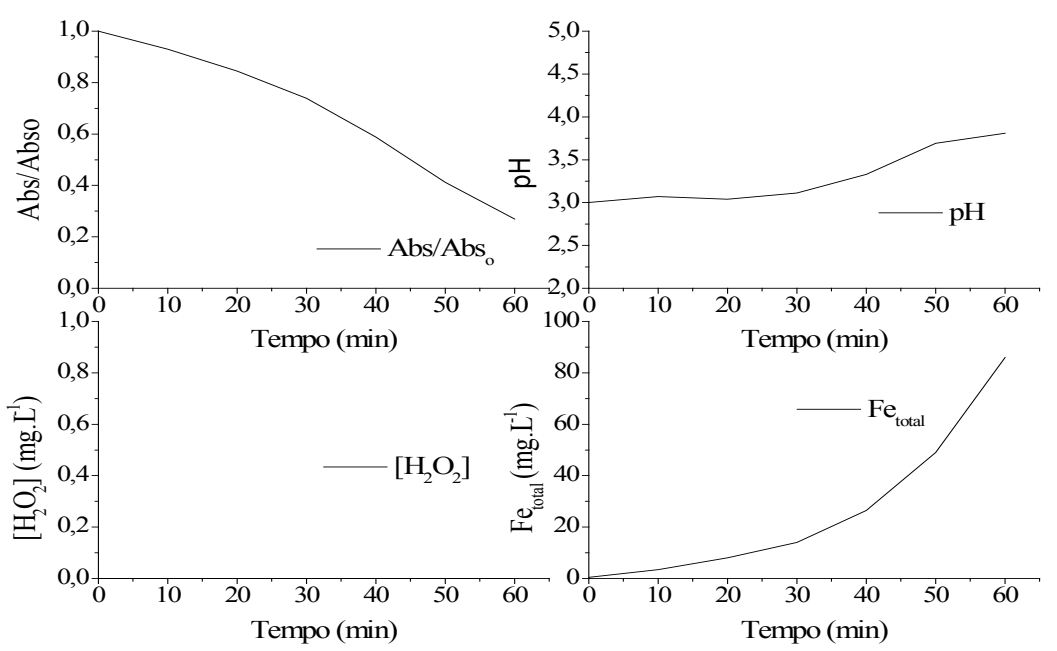

Figura 9. Perfil de degradação do corante, do pH, da concentração de peróxido de hidrogênio residual e da concentração de ferro total ao longo do tempo. Condição ensaiada: $\mathrm{Fe}=200 \mathrm{mg}$.L-1; [corante] = 20 mg.L-1; $\mathrm{pH}=3,0 ; \mathrm{T}=25^{\circ} \mathrm{C}$ e $150 \mathrm{rpm}$

linear para cada uma das retas, sendo 0,9848 e 0,9570 , respectivamente. Ainda assim foram determinadas as constantes cinéticas, cujos valores foram $1,96 \times 10^{-4}$ $\mathrm{s}^{-1}(\mathrm{pH}=2,0)$ e $2,66 \times 10^{-4} \mathrm{~s}^{-1}(\mathrm{pH}=3,0)$. Pelo perfil da Figura 10, é possível notar que a cinética de degradação do corante segue uma tendência de primeira ordem.

Foram realizados ensaios de degradação do corante em diferentes valores de temperatura, obedecendo as condições experimentais da Figura 8, com o propósito de obter os dados cinéticos suficientes para determinar a energia de ativação do sistema. Os resultados obtidos em diferentes temperaturas são apresentados na Figura 11.

Através da Figura 11, foi possível determinar a constante cinética para cada temperatura experimentada. A Tabela 1 apresenta os coeficientes de correlação e as constantes cinéticas para cada temperatura.

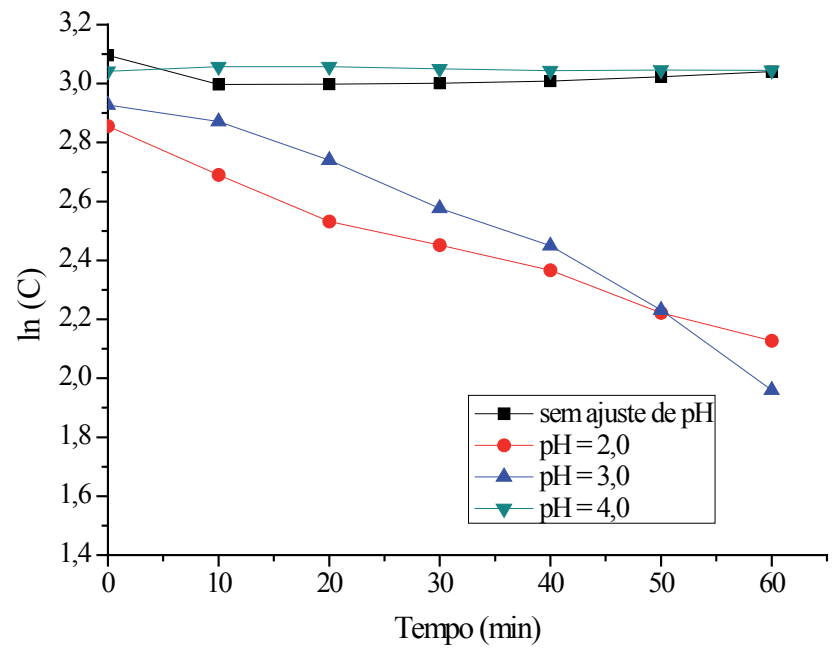

Figura 10. Perfil logaritmico da concentração do corante ao longo do tempo em diferentes valores de $\mathrm{pH}$. Condição ensaiada: $\mathrm{Fe}=200 \mathrm{mg} \cdot \mathrm{L}^{-1}$; [corante] $=20 \mathrm{mg} \cdot \mathrm{L}^{-1}$; $\mathrm{T}=25^{\circ} \mathrm{C}$ e $150 \mathrm{rpm}$ 


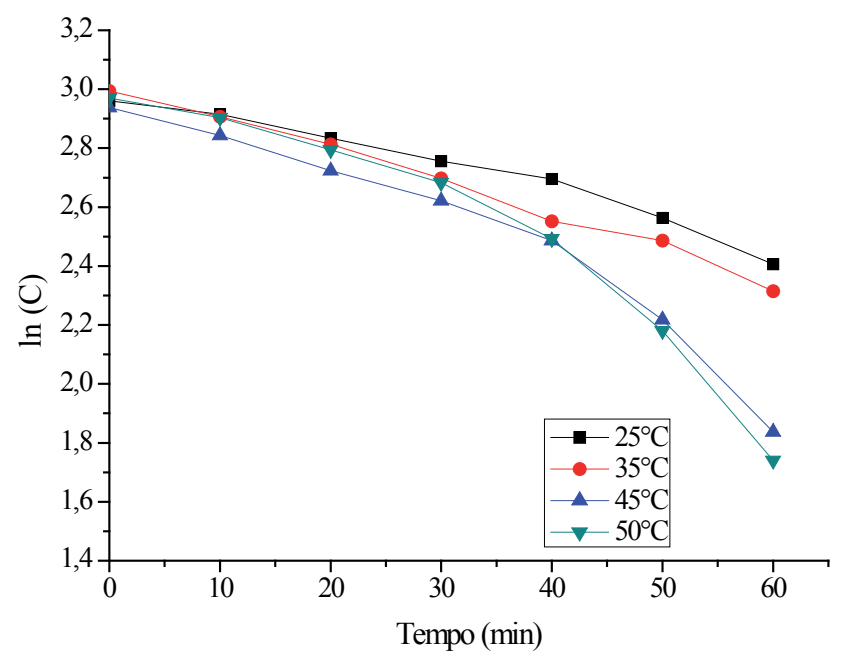

Figura 11. Perfil logarítmico da concentração do corante ao longo do tempo em diferentes valores de temperaturas. Condição ensaiada: $\mathrm{Fe}=200 \mathrm{mg}$.L-1; [corante] $=20$ mg.L-1; $\mathrm{pH}=3,0$ e $150 \mathrm{rpm}$.

Conforme já era esperado, o aumento da temperatura promove um decaimento mais acentuado na degradação do corante, e consequentemente, um aumento na constante cinétiva. Essa constatação foi comprovada por Arrhenius, através da equação característica da cinética de reações químicas, cujo o aumento da temperatura promove um aumento na constante cinética, e isso pode ser evidenciado pela Tabela 1, na qual foi apresentada a constante para cada faixa de temperatura ensaiada.

Os dados apresentados na Tabela 1 permitiram determinar a energia de ativação do sistema, através da Figura 12 no qual é observada o gráfico característico da equação de Arrhenius, cuja energia

Tabela 1. Equações de ajuste linear assim como os respectivos coeficientes de correlação e as constantes cinéticas para cada temperatura

\begin{tabular}{cccc}
\hline Temperatura $\left({ }^{\circ} \mathrm{C}\right)$ & Equação da reta & $\mathrm{R} 2$ & $\mathrm{k}(\mathrm{s}-1)$ \\
\hline 25 & $\mathrm{y}=-0,00895 \mathrm{x}+3,0013$ & 0,9557 & $1,49 \times 10-4$ \\
35 & $\mathrm{y}=-0,01122 \mathrm{x}+3,0171$ & 0,9888 & $1,87 \times 10-4$ \\
45 & $\mathrm{y}=-0,01711 \mathrm{x}+3,0372$ & 0,9057 & $2,85 \times 10-4$ \\
50 & $\mathrm{y}=-0,01942 \mathrm{x}+3,1201$ & 0,8798 & $3,24 \times 10-4$ \\
\hline
\end{tabular}

de ativação do sistema obtida foi de $25,8 \mathrm{~kJ} \cdot \mathrm{mol}^{-1}$, sugerindo assim, um mecanismo difusional, como a etapa controladora do sistema, pois segundo Ribeiro Filho (2005) e Schmal (2012), valores de energia de ativação menores que $30 \mathrm{~kJ}$, sugerem mecanismo difusional, enquanto que acima de $40 \mathrm{~kJ}$, indicam processos controlados pela reação química. E entre os valores mencionados pelos autores, são identificados como controle misto.

\section{Conclusão}

As nanopartículas de ferro de valência zero utilizadas apresentaram estrutura cúbica de corpo centrado e foram responsáveis pela degradação do corante em ambiente ácido. A melhor eficiência ocorreu em um ambiente ácido de $\mathrm{pH}$ igual a 3,0, em uma temperatura de $25^{\circ} \mathrm{C}$

As nZVI no valor de $\mathrm{pH}$ igual a 3,0, o mecanismo de degradação através da transferência de elétrons permitiu uma eficiência de degradação do corante para mais de uma hora de experimento, atingindo valores próximos de $80 \%$.

Não foi constatada a geração de peróxido de hidrogênio, através da metodologia usada, conforme foi sugerido por alguns autores. E somente metade da concentração de ferro total foi lixiviada, durante o período ensaiado.

Os ensaios realizados em diferentes temperaturas, permitiram sugerir ao sistema uma cinética de 


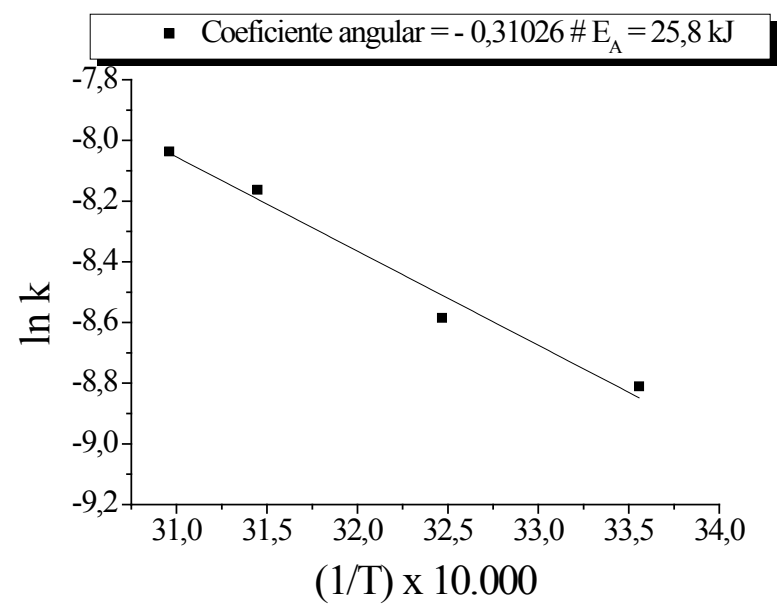

Figura 12. Gráfico de Arrhenius para o cálculo da energia de ativação do corante. Condição ensaiada: $\mathrm{Fe}=200 \mathrm{mg}$.L-1; [corante] = $20 \mathrm{mg}$.L-1; e $150 \mathrm{rpm}$

degradação de primeira ordem, e através das constantes cinéticas obtidas, para cada temperatura, foi possível determinar energia de ativação do processo, cujo valor foi igual a $25,8 \mathrm{~kJ}$, sugerindo assim um mecanismo difusional ao processo.

As nZVI se mostraram bastante promissoras ao serem utilizadas em processos de tratamento de efluentes, permitindo outros tipos de aplicação

\section{Referências}

Araújo, F. V. F. (2008).Estudo do processo Fenton Heterogêneo utilizando hematita $\left(\mathrm{Fe}_{2} \mathrm{O}_{3}\right)$ como catalisador na descoloração de soluções de corante reativo. Tese de Doutorado - Escola de Química, Universidade Federal do Rio de Janeiro, UFRJ, Rio de Janeiro.

Arruda, T. L. (2005). Uso de processos oxidativos avançados e ferro elementar na remediação de água subterrânea contendo compostos organoclorados. Dissertação de Mestrado, Instituto de Química, UNICAMP, Campinas, SP.

Braile, P. M. (1971). Despejos Industriais.Rio de Janeiro: Livraria Freitas Bastos.

Borba, C. E.;Módenes, A. N.; Espinoza-Quiñones, F. R.; Borba, F. H.;Bassi, A. F.; Ribeiro, C. (2012). Estudo da cinética e do equilíbrio de adsorção dos corantes azul turquesa $\mathrm{QG}$ e amarelo reativo $3 \mathrm{R}$ em carvão ativado. Engevista, 14 (2),135-142.

Carreira, M. F. (2006).Sistemas de Tratamento de Efluentes Têxteis - uma análise comparativa das tecnologias usadas no Brasil e na península Ibérica. Tese de Doutorado em Engenharia de Produção. Departamento de Engenharia de Produção. Universidade Federal de Santa Catarina, UFSC, Florianópolis.

Cavalcanti, J. E. W.A. (2009).Manual de Tratamento de Efluentes Industriais, $2^{\mathrm{a}}$ edição. São Paulo: Engenho Editora Técnica.

COLOUR INDEX.InternationalSocietyDyersandColoristsand American ofTextileChemistsandColorists - CD Room.

Elliot, D.W.; Zhang, W. X. Field assessment of nanoscaleBimetallic particles for groundwater treatment.Environ. Sci. Technol., 35,4922-4926.

Joo, S.;Feitz, A. J.; Waite, T. D. (2004).Oxidative Degradation of theCarbothioate Herbicide, Molinate, Using Nanoscale Zero-Valent Iron. Environ. Sci. Technol., 38, 2242-2247.

Karlsson, M. N. A.;Deppert, K.;Wacaser, B. A.;Karlsson, L. S.;Malm, J. -O. (2005)Size-controlled nanoparticles by thermal cracking of iron pentacarbonyl.App. Phys. A, 80, 1579-1583.

Keenan, C. R.;Sedlak, D. L. (2008).Factors Affecting the Yield ofOxidants from the Reaction ofNanoparticulate Zero-Valent Ironand Oxygen.Environ. Sci. Technol., 42, 262-1267.

Kim, B. H.; Park, C.; Kim, Y.; Jung, D.; Cho, H.; Park, S. H.; Ra, D.; Lee, D.; Jung, S. (2009). Characterization of zero valent iron prepared from byproduct of pickling line and its decomposition reaction activity.Korean J. Chem. Eng., 26, 1795-1799.

Lee, C.; Sedlak, D. L.(2008). Enhanced Formation of Oxidantsfrom Bimetallic Nickel-Iron Nanoparticles in the Presence of Oxygen.Environ. Sci. Technol.,42, 8528-8533.

Lin Y.; TsengH.; WeyM.;LinM.(2010).Characteristicsoftwotypesofstabilized nano zero-valentironandtransport in porous media. Science ofthe Total Environment, 408,2260-2267.

Marin, P.; Borba, C. E.;Módenes, A. P.; Oliveira, S. P. D.;Passaia, N.; Figueiredo, L. S. (2015). Avaliação do Efeito da temperatura, pH e granulometria do adsorvente na adsorção do corante azul reativo $5 \mathrm{G}$. Engevista, 17 (1), 59-68.

Mattos, I. L.; Shiraishi, K. A.; Braz, A. D.; Fernandes, J. R. (2003). Peróxido de Hidrogênio: Importância e determinação.Química Nova, 26 (3), 373-380.

METCALF \& EDDY. (2016).Tratamento de Efluentes e Recuperação de Recursos, $5^{\text {a }}$ ed.,Rio de Janeiro: McGraw Hill.

Módenes, A. N.; Espinoza, F. R.;Alflen, V. L.; Colombo, A.;Borba, C. E. (2011).Utilização da macrófitaegeria densa na biossorção do corante reativo 5G. Engevista, 13 (3), 160-166.

Moon, B.; Park, Y.; Park, K. (2011).Fenton oxidation of Orange II by prereduction using nanoscale zero-valent iron.Desalination, 268, 249-252.

NUNES, J. A. (2008).Tratamento Físico Químico de Águas Residuárias Industriais, $5^{\mathrm{a}}$ ed., Aracaju: Info Graphics Gráfica \& Editora.

Nurmi, J.;Tratnyek, P.;Sarathy, V.; Baer, D. R.;Amonette, J. E.;Pecher, K.; Wang, C.;Linehan, J. C.; Matson, D. W.; Penn, R. L.;Driessen, M. D. (2005). Characterization and Properties of Metallic Iron Nanoparticles: Spectroscopy, Electrochemistry, and Kinetics.Environ. Sci. Technol., 39, 1221-1230.

Oliveira, M. C.; Nogueira, R. F. P.; Gomes Neto, J. A.; Jardim, W. F.; Rohwedder, J. J. R. (2001). Sistema de Injeção em fluxo espectrofotométrico para monitorar peróxido de hidrogênio em processo de fotodegradação por reação foto-fenton.Química Nova, 24 (2), 188-190.

RibeiroFilho, F. A. (2005).Carbocloração do pentóxido de vanádio e aspectos do seucomportamentoquímico, 2005. Dissertaçãode Mestrado,Pont ifíciaUniversidadeCatólicado Rio de Janeiro, PUC-Rio.

Roine, A. (2006). "Outokumpu HSC-Chemistry 6.0, for Windows". Outokumpu Research Oy, Information Service, Helsinki, Finland.

Schmal, M. (2012).Cinética e Reatores: Aplicação à Engenharia Química teoria e exercícios, $2^{\mathrm{a}}$ edição, Rio de Janeiro: Synergia. 
Soeira, L. S. (2007).Estudo da degradação do p-nitrofenol por ferro de valência zero: avaliação de processos redutivos e oxidativos. Dissertação de Mestrado, Instituto de Química, UNICAMP, Campinas, SP.

Souza, M. G. O. (2007).Utilização de Nanopartículas de Ferro na Remediação de Águas Subterrâneas Contaminadas por Compostos Orgânicos. Dissertação de Mestrado,COPPE, Universidade Federal do Rio de Janeiro, UFRJ, Rio de Janeiro.

Sun, Y.; Li, X.; Cao, J.; Zhang, W.; Wang, H. P. (2006)Characterization of zero-valent iron nanoparticles.Adv. Colloid and Interf. Sci., 120, 47-56.

Sun, Y.; Li, X.; Zhang, W.; Wang, H. (2007).A method for the preparation of stable dispersion of zero-valent iron nanoparticles.Colloids and Surf. A, 308, 60-66.

Sun, X; Yu, H; Da Zhenga; Wang, X; LI, J; Wang, L. (2013). Incorporation of nanoscale zero-valent iron particles inside the channels of SBA-15 silica rods by a "two solvents" reduction technique.Applied Surface Science, 279, $1-6$.

Xia, L.; Gu Z.; Zhang, Z.; Zhang, J.; Hermanowicz, S. W. (2014).Removal of chloramphenicol from aqueous solution by nanoscale zero-valent iron particles, Chemical Engineering Journal, 257, 98-104.

Zhang, H.; Wang, Y.; Tao, G.; Chai, Y.;Que, G. (2011).Chemical Synthesis of $\mathrm{Fe}$ Nanocrystals via Hydrogenation of Ferric Acetylacetonate, In: Materials for Renewable Energy \& Environment (ICMREE), International Conference, 20-22 May. 2011, Shanghai, IEEEXplore Digital Library: vol.2, 2066-2070. 